\title{
Trauma Craniano Violento (TCV): Relação entre Conhecimento de Pais, Potencial de Abuso Infantil e Status Socioeconômico Familiar
}

\author{
Ana Paula Soares** (D, Sabrina Mazo d'Affonseca (D, \& Rachel de Faria Brino (D \\ Universidade Federal de São Carlos, São Carlos, SP, Brasil
}

\begin{abstract}
RESUMO - Descreve a relação entre conhecimento sobre Trauma Craniano Violento (TCV), status socioeconômico e potencial de maus-tratos infantis. Dezenove pais e 61 mães responderam aos instrumentos Escala de Atitudes Frente ao Choro do Bebê, Inventário de Potencial de Abuso Infantil e Questionário Socioeconômico. A correlação de Spearman demonstrou resultados significativos entre escolaridade e renda com crenças sobre cuidados com o bebê $(r=0,32, p=$ 0,$004 ; \mathrm{r}=0,22, \mathrm{p}=0,05$, respectivamente), poder aquisitivo e escolaridade com conhecimentos sobre choro infantil $(\mathrm{r}=$ $-0,40, p<0,001 ; r=-0,22, p=0,05$, respectivamente), número de filhos com estratégias para lidar com choro $(r=-0,29$, $p=0,01)$ e rigidez com consequências de sacudir o bebê e crenças sobre cuidados com o bebê $(r=-0,29, p=0,008 ; r=$ $-0,359, \mathrm{p}=0,001$, respectivamente). Considera importante direcionar intervenções às necessidades de cada população e trabalhar a flexibilidade parental para reduzir o TCV.
\end{abstract}

PALAVRAS-CHAVE: Trauma Craniano Violento, abuso infantil, família, classe socioeconômica

\section{Abusive Head Trauma (AHT): Relationship between Knowledge of Parents, Child Abuse Potential and Family Socioeconomic Status}

\begin{abstract}
Describes the relationship between knowledge on Abusive Head Trauma, socioeconomic status and child abuse potential. 19 parents and 61 mothers filled out the following instruments: Attitudes Towards Infant Crying Scale, Child Abuse Potential Inventory and Socioeconomic Questionnaire. Spearman's correlation found significant results: schooling and income showed correlation with beliefs about infant care $(r=0,32, p=0,004 ; r=0,22, p=0,05$ respectively), purchasing power and schooling correlated with knowledge on infant crying $(r=-0,40, p<0,001 ; r=-0,22, p=0,05$ respectively), number of children correlated with strategies for coping with baby crying $(r=-0,29, p=0,01)$ and rigidity with consequences of shaking a baby and beliefs $(r=-0,29, p=0,008 ; r=-0,36, p=0,001$ respectively). It is important to target interventions to the needs of each population and to work with parental flexibility to reduce the incidence of AHT.
\end{abstract}

KEYWORDS: Abusive Head Trauma, child abuse, family, socioeconomic status

O Trauma Craniano Violento (TCV) é consequência de comportamentos violentos perpetrados, comumente, por pais e/ou cuidadores contra crianças pequenas (Kemp, 2011). Tais comportamentos envolvem a sacudida violenta do bebê ou o impacto brusco intencional de sua cabeça contra uma superfície sólida, resultando em ferimento craniano ou intracraniano (Lopes et al., 2013; Parks et al., 2012). A população mais vulnerável a este tipo de violência são crianças menores de 3 anos (Harden et al., 2016), do sexo masculino (Sieswerda-Hoogendoorn et al., 2013) e que apresentam déficits de desenvolvimento (Yaylaci et al., 2016).

Outros fatores de risco também estão relacionados ao TCV, de acordo com a literatura. Entre eles, estão o baixo nível socioeconômico das famílias, refletido em baixa escolaridade, residência em bairros mais pobres, com alto índice de criminalidade, alta taxa de desemprego e menor renda (Fujiwara et al., 2016; Yaylaci et al., 2016); baixa idade parental (Kelly et al., 2017); altos níveis de estresse na família (Selassie et al., 2013; Yamada \& Fujiwara,

\footnotetext{
* O referido artigo é baseado na Dissertação de Mestrado da primeira autora.

** E-mail: apmasoares@gmail.com

n Submetido: 27/11/2019; Revisado: 21/05/2020; Aceito: 17/06/2020.
} 
2014); com histórico de violência doméstica e contra outras crianças da casa (Isumi \& Fujiwara, 2016; Yaylaci et al., 2016); histórico de abuso de substâncias (SieswerdaHoogendoorn et al., 2013); presença de doenças mentais (Sieswerda-Hoogendoorn et al., 2013); e doenças físicas nos pais (Yaylaci et al., 2016); além de pouca experiência ou conhecimentos relativos aos cuidados e desenvolvimento infantil pelos cuidadores (Barr, 2014).

Apesar de todos esses fatores de risco, Tanoue e Matsui (2015), comparando amostras de crianças que sofreram o TCV e crianças que sofreram outros tipos de abuso, verificaram que os fatores de risco estão menos presentes no TCV do que em outros tipos de violência. Isso porque o principal gatilho do comportamento de sacudir a criança é o choro que ocorre de forma prolongada, imprevisível, inexplicável e de difícil controle (Barr, 2014).

Nesse sentido, Barr (2014) aponta que essas características do choro colaboram para o aumento do estresse, frustração, raiva e sensação de falta de habilidade para acalmar o bebê. Assim, cuidar de um bebê pode ser considerada uma tarefa cansativa e estressante, já que $73 \%$ em uma amostra de 90 pais brasileiros concordaram com esta afirmativa (Lopes \& Williams, 2016). Segundo Barr (2014), é possível que os pais experimentem diversas tentativas e técnicas para que o bebê pare de chorar e, quando não têm o resultado esperado, esses sentimentos negativos aumentem e, com eles, a probabilidade de sacudir a criança. Quando sacudida, a criança geralmente cessa o choro, o que faz com que essa prática se repita no futuro, por ter sido eficaz em seu propósito (Barr, 2014).

Sacudir a criança, no entanto, pode ter consequências negativas para sua saúde e desenvolvimento, com sequelas neurológicas, físicas, cognitivas e comportamentais, dentre elas, déficits motores, visuais, auditivos e de fala, dificuldades de aprendizagem, convulsões, paralisia cerebral, distúrbios comportamentais e de sono, além de morte (Chevignard \& Lind, 2014; Kemp, 2011; Lopes et al., 2013; Nuño et al., 2015). Conforme descrito por Chevingnard e Lind (2014) em sua revisão de literatura, devido ao exposto, essas crianças tendem a necessitar de longo período de hospitalização, além de cuidados médicos, reabilitação física, ocupacional, psicomotora, psicológica e de linguagem a longo prazo, educação especial e intervenções de suporte psicológico, orientações e informações direcionadas à família, ocasionando, portanto, grandes custos financeiros.

Nesse sentido, o planejamento de programas de prevenção direcionados a situações de risco podem constituir importantes ferramentas para a redução das taxas de TCV (Lopes et al., 2013). Para isso, são necessárias investigações a respeito de fatores de risco presentes na população (Lopes et al., 2013). Ao mesmo tempo, a vulnerabilidade socioeconômica é importante fator que aumenta a probabilidade de ocorrerem vários tipos de violência (Molnar et al., 2016), incluindo o TCV, muitas vezes porque as famílias possuem menor acesso a serviços de saúde se comparadas com famílias de maior poder aquisitivo (Nuño et al., 2015).

No entanto, algumas pesquisas apresentam dados divergentes. Na França, por exemplo, um estudo retrospectivo utilizando dados judiciais enfatiza que os principais perpetradores desse tipo de violência foram o pai, a mãe ou ambos, que - diferentemente do que afirmam outros autores - em sua maioria, viviam como casal (apenas 6\% das mães viviam sozinhas e $14 \%$ sem a presença do pai biológico da criança), apresentavam alto grau de instrução (com $46 \%$ das mães possuindo ensino superior completo) e eram empregados (Tursz \& Cook, 2014).

Sendo assim, o objetivo deste estudo é investigar se, em uma amostra de pais de um município de médio porte do interior de São Paulo, houve relação entre o conhecimento de pais sobre o Trauma Craniano Violento, o status socioeconômico da família e o potencial de maus-tratos desses em relação aos seus filhos.

\section{MÉTODO}

A seguir, é apresentado o método aplicado na pesquisa, incluindo os participantes que compuseram a amostra, o local e os instrumentos de coleta de dados, o tipo de pesquisa e os procedimentos de coleta e análise de dados.

\section{Participantes}

Participaram da pesquisa 61 mães e 19 pais de crianças recém-nascidas ou em gestação e de um ou mais filhos entre 2 e 12 anos. Cabe a ressalva de que ter filhos ente 2 e 12 anos é critério para a aplicação de um dos instrumentos utilizados, o Inventário de Potencial de Abuso Infantil (CAP), uma vez que este exige que as respostas sejam dadas considerando uma criança dessa faixa etária, de forma não hipotética (Rios et al., 2013). Essas mães estavam internadas nas enfermarias da maternidade no período gravídico ou após o parto, e os pais que participaram as acompanhavam. É importante dizer, também, que $36,84 \%$ dos homens ( 7 dos 19) não eram pais biológicos das crianças entre 2 e 12 anos, no entanto, as criavam junto com as mães há, pelo menos, dois anos. O tempo de gestação entre as mulheres grávidas era de 32,77 semanas (DP =9,17). Esta amostra constituiu-se por conveniência, isto é, os participantes foram selecionados dentro da maternidade a partir dos critérios mencionados acima e de acordo com o seu interesse em participar. 


\section{Instrumentos}

A coleta de dados foi realizada com o uso de três instrumentos:

1) Escala de Atitudes Frente ao Choro do Bebê - instrumento elaborado por Lopes (2017) para avaliar conhecimentos sobre o TCV e é composto por seis fatores. $\mathrm{O}$ primeiro deles diz respeito às Consequências de sacudir $o$ bebê (10 itens), com itens descrevendo consequências negativas de sacudir a criança; o segundo, às Estratégias para o bem-estar do cuidador (12 itens), com itens direcionados a fazer o cuidador se sentir bem ao cuidar do bebê; o terceiro fator concerne às Estratégias para lidar com o choro relacionadas ao bebê (11 itens), ou seja, estratégias que os cuidadores utilizam para acalmar o bebê e o fazer parar de chorar; o quarto, às Estratégias para lidar com o choro relacionadas ao cuidador (4 itens), descrevendo estratégias que o cuidador utiliza para lidar com seus próprios sentimentos em relação ao choro do bebê; o quinto fator é relativo a Crenças sobre o cuidado do bebê (7 itens) e é composto por sentenças que indicam crenças adequadas e inadequadas sobre o cuidado com a criança; e, por fim, o sexto abrange Características do choro (6 itens), detalhando características do choro nos primeiros meses de vida. O instrumento se inicia apresentando perguntas relativas às características socioeconômicas dos respondentes, como a renda bruta familiar mensal e o número de anos de estudo, e segue com 50 itens com os quais o participante deve Discordar Totalmente (1), Discordar (2), Concordar (3) e Concordar Totalmente (4) caracterizando-se, portanto, como uma escala do tipo Likert;

2) Inventário de Potencial de Abuso Infantil (CAP) resultado da tradução e adaptação transcultural para o Brasil do Child Abuse Potencial Inventory realizada por Rios et al. (2013). Trata-se de um instrumento composto por 160 itens, com os quais o respondente deve concordar (C) ou discordar (D). O CAP é composto por fatores que medem: o grau de sofrimento, com padrões de sentir-se frustrado, triste, preocupado, com medo, solitário, rejeitado, com raiva e confuso; rigidez, que descreve a inflexibilidade em relação à aparência e comportamento da criança; infelicidade, que mede a polaridade entre felicidade e infelicidade com itens que avaliam se a pessoa se considera feliz e sortuda, por exemplo; problemas com a criança e consigo, com itens que verificam se a criança é avaliada pelo adulto de forma negativa e se este considera que possui problemas físicos; problemas com a família, identificando se há dificuldades de relacionamento com a família; e problemas com os outros, onde os outros são vistos como causas de decepções e sofrimento, de forma que essas relações não se constituem como recursos e sim como problemas;

3) Questionário socioeconômico - baseado nos Critérios de Classificação Econômica do Brasil (CCEB) da Associação Brasileira de Empresas e Pesquisa (ABEP) de 2014. O CCBE da ABEP de 2014 considera para a classificação de classes econômicas (A, B1, B2, C1, C2, $\mathrm{D}$ e E) a posse e a quantidade de itens, como televisão em cores, rádio, banheiro, automóvel, máquina de lavar, vídeo cassete e/ou DVD, geladeira e freezer, além de empregada mensalista e o grau de instrução. Esse instrumento tem por objetivo investigar as características socioeconômicas dos participantes.

\section{Tipo de pesquisa}

Trata-se de uma pesquisa de levantamento, utilizando o método quantitativo.

\section{Procedimentos de coleta de dados}

A coleta de dados foi feita nas dependências de uma maternidade de um município de médio porte do interior do estado de São Paulo.

Primeiramente, o projeto foi submetido para a avaliação das comissões de ética de duas instituições (a maternidade e a Secretaria de Saúde do município). Em um primeiro momento, apenas a maternidade havia sido contatada; no entanto, passados cinco meses, a autorização para a realização da coleta ainda não havia sido disponibilizada. Desta forma, optou-se por submeter o projeto também à Secretaria de Saúde, com o objetivo de recrutar participantes nas Unidades Básicas de Saúde (UBS's). Ao final, ambas as instituições retornaram com resposta positiva no mesmo período, fornecendo uma carta de aceite. Em seguida, o projeto foi encaminhado ao Comitê de Ética e Pesquisa (CEP) da Universidade pela Plataforma Brasil, onde foi avaliado e autorizado (CAEE: 77746417.1.0000.5504).

Posteriormente, foi feito contato com o médico e enfermeira chefes da maternidade, a fim de esclarecer os objetivos e procedimentos da pesquisa, assim como definir dias e horários para a coleta. Como as enfermarias da maternidade supriram o número de participantes, não foi necessário recorrer às UBS's. Em sequência, houve uma breve conversa com as enfermeiras responsáveis pelas enfermarias de gestantes e puérperas com a finalidade de conhecer o funcionamento e rotina de trabalho e de aprender a manusear os prontuários e ler os quadros fixados com as informações das pacientes internadas para a identificação dos leitos onde se encontrava a população alvo do estudo. No entanto, como os prontuários geralmente estavam sendo utilizados pela equipe de saúde e, portanto, estavam indisponíveis, e os quadros muitas vezes se encontravam desatualizados, a abordagem dos participantes foi feita leito a leito.

Dessa forma, as pacientes e seus companheiros, pais de seus bebês, quando presentes, eram abordados em seus 
leitos e nos corredores. A pesquisadora, então, se apresentava e fazia perguntas concernentes aos critérios de inclusão e exclusão: se estavam gestantes ou em pós-parto e se havia outros filhos vivos e quais eram suas idades. Quando os pais não tinham outros filhos ou quando suas idades não correspondiam à faixa-etária pretendida ( 2 a 12 anos), era explicado que não poderiam participar da pesquisa, mas que havia disponibilidade para conversar e tirar dúvidas a respeito do TCV. Aos pais que atendiam aos critérios, eram esclarecidos os objetivos e procedimentos da pesquisa e garantida a voluntariedade da participação.

Aumentar a adesão destes pais para responder às escalas e questionários, no entanto, não foi tarefa fácil. Em um primeiro momento, o projeto era apresentado com o objetivo de verificar situações de violência dos pais com o bebê e outras crianças. Era perceptível que a palavra 'violência' afugentava os possíveis participantes. Dessa forma, o discurso de apresentação do projeto foi modificado junto com o TCLE - Termo de Consentimento Livre e Esclarecido - e passou a mostrar que o objetivo era verificar de que forma a maneira como os pais cuidam das crianças quando pequenas influencia na sua saúde e desenvolvimento posterior. Outra dificuldade encontrada para a adesão foi o número de itens a serem respondidos, totalizando 210 se somados os 50 itens da Escala de Atitudes Frente ao Choro do Bebê e 160 do CAP. Para que os participantes ficassem menos assustados, era informado o tempo médio que levava para responder a todos os instrumentos: entre 40 e 60 minutos.

Àqueles que concordavam em colaborar respondendo aos instrumentos, era dado o TCLE. Este poderia ser lido pelas mães e/ou pais ou, quando solicitado por eles, pela pesquisadora em voz alta. Ao término da leitura, as dúvidas, quando existiam, eram sanadas; os termos eram assinados pela pesquisadora e participantes; e, então, tinha início a coleta de dados, começando pela Escala de Atitudes Frente ao Choro do bebê, seguida pelo CAP e finalizando com o Critério Brasil. Esses instrumentos foram aplicados pela primeira autora que contou com treinamento prévio para tal.

A aplicação dos instrumentos era feita em forma de entrevista, individualmente, com um participante respondendo por vez. Como a coleta foi feita nas dependências da maternidade, em corredores e enfermarias, não havendo sala reservada para este fim, era frequente a presença de outras pessoas, usuários internados e profissionais de saúde por perto durante a aplicação dos instrumentos. Para minimizar desconfortos, era oferecida a possibilidade de leitura dos itens pelo participante em voz baixa, desde que o mesmo respondesse em voz alta para que a pesquisadora marcasse no instrumento. Também era assegurado ao participante que ele não precisaria responder aos itens com os quais não se sentisse confortável.
Ao término de cada coleta, os participantes recebiam uma devolutiva dos dados coletados com uma breve explicação sobre o que é o Trauma Craniano Violento pediátrico, quais comportamentos podem causá-lo, quais são as possíveis consequências negativas para a saúde e desenvolvimento da criança, assim como sobre a importância da identificação e manejo de situações de estresse no momento dos cuidados com o bebê. Ao todo, a coleta de dados durou cerca de cinco meses e era feita às segundas, quartas e sextas-feiras, das $14 \mathrm{~h}$ às $17 \mathrm{~h}$. $O$ período de coleta de dados teve o seu início no dia 20 de novembro de 2017 e término em 14 de abril de 2018 .

\section{Procedimento de análise de dados}

No que diz respeito aos dados advindos do Questionário Socioeconômico, foram calculados média e desvio padrão para variáveis como renda familiar mensal, número de filhos, escolaridade em anos, assim como a porcentagem da via do atendimento de saúde e ocupação dos participantes. Para a Escala de Atitudes Frente ao Choro do Bebê, foi feita a soma das pontuações dos itens de cada fator e, em seguida, o resultado da soma foi dividido pelo número de itens que compunham os fatores. Os dados obtidos com a aplicação do CAP foram analisados de acordo com as instruções da tabela de análise fornecida pelos autores do instrumento (Rios, 2010).

Também foram calculadas as correlações entre as variáveis socioeconômicas (de escolaridade em anos, número de filhos, renda mensal familiar bruta declarada e escore no Critério Brasil), as subescalas da Escala de Atitudes Frente ao Choro do Bebê e as subescalas do CAP. As correlações foram calculadas com o teste estatístico não paramétrico de correlação de Spearman por meio do software SPSS. Considera-se p-valor menor ou igual a 0,05 .

Além disso, para mensurar a confiabilidade dos instrumentos, foram calculados os Coeficientes KR20 para escalas dicotômicas do questionário CAP (Kuder \& Richardson, 1937) e Lambda 2 de Guttman para as componentes da Escala de Atitudes Frente ao Choro do Bebê (Guttman, 1945). Em ambas as medidas, os valores aceitáveis são àqueles acima de 0,70 , sendo que quanto mais próximo de 1 , maior a confiabilidade do instrumento. A Tabela 1 descreve os valores de confiabilidade para as subescalas da Escala de Atitudes Frente ao Choro do Bebê e do Questionário CAP.

Para calcular o intervalo de confiança de $95 \%$ do coeficiente de correlação de Spearman foi utilizado o método empírico de Boostrapping (Efrom, 1980), sendo consideradas 10 mil reamostras de Boostrapping para este estudo. 
Tabela 1

Confiabilidade por subescala

\begin{tabular}{lccccc}
\hline & Número de itens & $\begin{array}{c}\text { Lambda 2 de } \\
\text { Guttman(1945) }\end{array}$ & Número de itens & $\begin{array}{c}\text { Kuder-Richardson } \\
\mathbf{2 0}(\mathbf{1 9 3 7})\end{array}$ \\
\hline Consequências Sacudir & 10 & 0,883 & Abuso & 77 & 0,910 \\
Bem-estar cuidador & 12 & 0,780 & Sofrimento & 36 & 0,927 \\
Estratégia bebê & 11 & 0,740 & Rigidez & 14 & 0,765 \\
Estratégia cuidador & 4 & 0,551 & Infelicidade & 11 & 0,304 \\
Crenças & 7 & 0,567 & Criança & 6 & $-0,348$ \\
Características do choro & 6 & 0,405 & Família & 4 & 0,630 \\
& & & Outros & 6 & 0,423 \\
\hline
\end{tabular}

\section{RESULTADOS}

A Tabela 2 descreve a caracterização da amostra em média e desvio padrão das variáveis "número de filhos" $\left(\mathrm{N}^{\mathrm{o}} \mathrm{de}\right.$ filhos) e Escolaridade (em anos). Também foram descritas as porcentagens das variáveis "renda", dividida pornúmero de Salários Mínimos (SM) recebidos, "ocupação", dividida em do lar, ocupação remunerada e estudante, e"Atendimento de Saúde" (At. Saúde), sendo este por meio do Sistema Único de Saúde (SUS), plano de saúde ou particular.

De acordo com a tabela, trata-se de uma amostra cuja renda é majoritariamente de um a três salários mínimos $(61,25 \%)$, com escolaridade média de 10,58 anos (DP =2,55), o que corresponde ao Ensino Médio incompleto, sendo uma população majoritariamente atendida pelo SUS $(77,50 \%)$. A principal ocupação relatada foi a remunerada $(57,50 \%)$, e o número médio de filhos era de $1,27(\mathrm{DP}=1,42)$.

A Tabela 3 descreve as correlações entre as variáveis socioeconômicas "Escolaridade" (em anos), "Escore no Critério Brasil", "Renda" e "Número de Filhos" $\left(\mathrm{N}^{\circ}\right.$ de Filhos) e as subescalas na Escala de Atitudes Frente ao Choro do Bebê com o coeficiente de correlação, significância e intervalo de confiança. Estão sinalizados com asterisco os coeficientes que indicam correlação significativa.

Como mostra a tabela, em relação às variáveis sociodemográficas e às subescalas da Escala de Atitudes Frente ao Choro do Bebê, a escolaridade e a renda têm correlação significativa e positiva com as crenças sobre o cuidado do bebê $(r=0,32, p=0,004 ; r=0,22, p=0,05$, respectivamente). Assim, possivelmente estes dados indicam que maior escolaridade e renda podem estar relacionadas a crenças mais adequadas a respeito dos cuidados com o bebê. Por outro lado, o escore no Critério Brasil e a escolaridade foram significativa e negativamente correlacionados às características do choro $(\mathrm{r}=-0,40, \mathrm{p}<0,001 ; \mathrm{r}=-0,22$, $\mathrm{p}=0,05$, respectivamente); ou seja, é possível indicar que maior escolaridade e poder de compra podem estar associados a menores conhecimentos sobre características do choro infantil. Além disso, foi verificada uma correlação significativa e negativa entre o número de filhos e as estratégias para lidar com o choro da criança relacionadas ao cuidador $(\mathrm{r}=-0,29, \mathrm{p}=0,01)$, o que dá indícios de que a maior quantidade de filhos pode levar a dificuldades na utilização de estratégias direcionadas para acalmar o cuidador quando o bebê está chorando.

A Tabela 4, por sua vez, descreve as correlações entre as subescalas do inventário CAP e as subescalas da Escala de Atitudes Frente ao Choro do Bebê com o coeficiente de correlação, a significância e o intervalo de confiança. Estão sinalizados com asterisco os coeficientes que indicam correlação significativa.

No que diz respeito às subescalas do CAP, a de Rigidez foi a que teve maior número de correlações significativas em relação às subescalas da Escala de Atitudes Frente ao Choro, estando relacionada negativamente às consequências de sacudir o bebê $(r=-0,30, p=0,009)$, às crenças sobre o cuidado do bebê $(r=-0,36, p=0,001)$ e ao escore total na escala $(r=-0,30, p=0,007)$. Assim, é possível que a rigidez, ou seja, a inflexibilidade em relação à aparência e ao comportamento da criança, possa estar relacionada a menores conhecimentos em relação às consequências negativas de sacudir o bebê, a crenças inadequadas sobre os cuidados com a criança e ao escore total mais baixo da Escala de Atitudes Frente ao Choro do bebê. Além da subescala de Rigidez, a de Infelicidade obteve correlação significativa e positiva com o conhecimento sobre as características do choro $(r=0,232, p=0,04)$, isto é, quanto maior o escore na subescala de infelicidade, também é maior o escore da subescala de conhecimentos relativos às características do choro. 
Tabela 2

Caracterização da Amostra

\begin{tabular}{|c|c|c|c|c|c|}
\hline & & Média & DP & $\%$ & $\mathbf{N}^{\circ}$ Bruto \\
\hline \multirow[t]{4}{*}{ Renda (R\$) } & Até $1 \mathrm{SM}$ & & & 17,50 & \\
\hline & 1 até $3 \mathrm{SM}$ & & & 61,25 & \\
\hline & 3 até $7 \mathrm{SM}$ & & & 21,25 & \\
\hline & Mais que $7 \mathrm{SM}$ & & & 3,75 & \\
\hline Escolaridade (anos) & & 10,58 & 2,55 & & \\
\hline $\mathrm{N}^{\circ}$ de filhos & & 1,27 & 1,42 & & \\
\hline \multirow[t]{3}{*}{ Ocupação } & Do lar & & & 41,25 & 33 \\
\hline & $\begin{array}{l}\text { Ocupação } \\
\text { Remunerada }\end{array}$ & & & 57,50 & 46 \\
\hline & Estudante & & & 1,25 & 1 \\
\hline \multirow[t]{3}{*}{ Atendimento Saúde } & Particular & & & 7,50 & 6 \\
\hline & Plano de Saúde & & & 15,00 & 12 \\
\hline & SUS & & & 77,50 & 62 \\
\hline
\end{tabular}

Tabela 3

Correlação entre Variáveis Socioeconômicas e Escala de Atitudes Frente ao Choro do Bebê

\begin{tabular}{lcccccccc}
\hline & \multicolumn{2}{c}{$\mathbf{N}^{\circ}$ de filhos } & \multicolumn{2}{c}{ Escolaridade } & \multicolumn{2}{c}{ Renda } & \multicolumn{2}{c}{ Escore CB } \\
\hline & $\mathbf{R}$ & $\begin{array}{c}\text { Intervalo de } \\
\text { confiança }\end{array}$ & $\mathbf{r}$ & $\begin{array}{c}\text { Intervalo de } \\
\text { confiança }\end{array}$ & $\mathbf{r}$ & $\begin{array}{c}\text { Intervalo de } \\
\text { confiança }\end{array}$ & $\begin{array}{c}\text { Intervalo de } \\
\text { confiança }\end{array}$ \\
\hline Consequências sacudir & $-0,06$ & $(-0,28 ; 0,16)$ & 0,16 & $(-0,06 ; 0,37)$ & $-0,03$ & $(-0,25 ; 0,19)$ & 0,06 & $(-0,17 ; 0,27)$ \\
Bem-estar cuidador & $-0,02$ & $(-0,24 ; 0,19)$ & 0,04 & $(-0,17 ; 0,24)$ & 0,11 & $(-0,13 ; 0,34)$ & $-0,06$ & $(-0,29 ; 0,18)$ \\
Estratégia bebê & $-0,07$ & $(-0,27 ; 0,15)$ & $-0,02$ & $(-0,24 ; 0,20)$ & $-0,14$ & $(-0,36 ; 0,09)$ & $-0,07$ & $(-0,29 ; 0,16)$ \\
Estratégia cuidador & $-0,29^{* *}$ & $(-0,48 ;-0,07)$ & 0,09 & $(-0,13 ; 0,31)$ & $-0,03$ & $(-0,24 ; 0,20)$ & $-0,02$ & $(-0,24 ; 0,20)$ \\
Crenças & $-0,06$ & $(-0,28 ; 0,17)$ & $0,32^{*}$ & $(0,11 ; 0,51)$ & $0,22 *$ & $(0,01 ; 0,42)$ & 0,21 & $(-0,02 ; 0,42)$ \\
Características do choro & 0,07 & $(-0,14 ; 0,27)$ & $-0,22^{*}$ & $(-0,42 ;-0,00)$ & $-0,17$ & $(-0,37 ; 0,04)$ & $-0,39 * *$ & $(-0,56 ;-0,20)$ \\
Total & $-0,01$. & $(-0,31 ; 0,12)$ & 0,18 & $(-0,04 ; 0,38)$ & 0,04 & $(-0,18 ; 0,26)$ & $-0,09$ & $(-0,31 ; 0,14)$ \\
\hline
\end{tabular}

Nota: ${ }^{*} \mathrm{p}<0,05 ; * * \mathrm{p}<0,01$

Finalmente, a Tabela 5 descreve as correlações entre as variáveis socioeconômicas e as subescalas do inventário CAP com o coeficiente de correlação, significância e intervalo de confiança. Estão sinalizados com asterisco os coeficientes que indicam correlação significativa.

As variáveis sociodemográficas obtiveram correlação significativa com as subescalas de sofrimento, rigidez, infelicidade e problemas com a família. A escolaridade está negativamente correlacionada ao escore total da escala $(r=$ $-0,29, \mathrm{p}=0,010)$, e à subescala de Sofrimento $(\mathrm{r}=-0,27, \mathrm{p}=$ $0,016)$ e Rigidez $(r=-0,26, p=0,019)$. O escore no Critério Brasil se correlacionou também negativamente com o escore total da escala $(r=-47, p<0,001)$ e as subescalas Sofrimento $(r=-0,49, p<0,001)$, Infelicidade $(r=-0,30, p=0,007)$ e Problemas com os outros $(r=-0,23, p=0,038)$. Por último, a renda obteve correlação negativa com o escore total da escala $(r=-0,44, p<0,001)$ e as subescalas de Sofrimento $(\mathrm{r}=-0,47, \mathrm{p}<0,001)$, Infelicidade $(\mathrm{r}=-0,31, \mathrm{p}=0,005) \mathrm{e}$ Problemas com a família $(\mathrm{r}=-0,24, \mathrm{p}=0,035)$.

Assim, as correlações indicam que é possível que haja maior exposição de populações com menor renda, escolaridade e poder aquisitivo a situações de abuso, sofrimento, infelicidade, problemas com a família, com a criança e com os outros. 

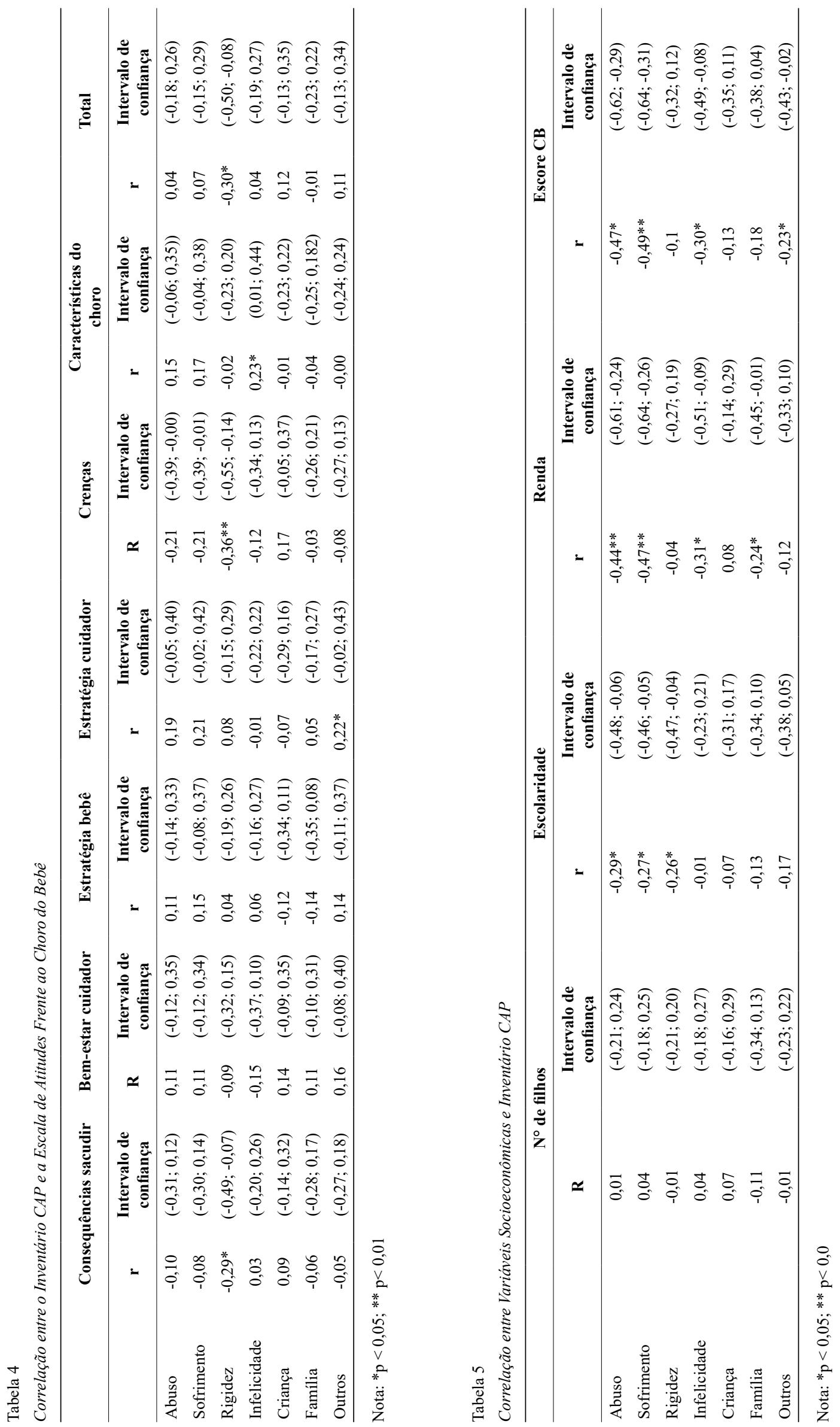


\section{DISCUSSÃO}

Durante a análise de dados, foram encontradas correlações entre variáveis que indicavam o nível socioeconômico mais baixo, como menor escolaridade, renda e poder aquisitivo, com maior exposição a situações de violência, sofrimento, infelicidade, problemas com a criança, consigo e com os outros. Nesse sentido, a literatura vem corroborando estes dados: segundo Leetch et al. (2015), os riscos de acontecer abuso infantil na convivência familiar aumentam quando os pais estão desempregados e possuem baixo status socioeconômico. Este último pode ser mensurado pela baixa renda e baixa escolaridade, aumentando de cinco a sete vezes a probabilidade de ocorrência de maus-tratos contra a criança se comparado com pais de maior renda e escolaridade (Leetch et al., 2015).

No Brasil, Piñón (2008), ao analisar uma amostra de 433 participantes, observou que houve correlação negativa das variáveis escolaridade e classe econômica com o escore total obtido no CAP, sugerindo que o potencial de risco para maus-tratos aumenta em populações de menor nível socioeconômico e grau de instrução.

Especificamente no TCV, foram encontradas correlações indicando que o nível socioeconômico mais baixo, dado pela renda e escolaridade menor, está relacionado a crenças mais inadequadas em relação aos cuidados do bebê, sendo um fator que aumenta a probabilidade de ocorrer esse tipo de maus-tratos. Alguns estudos publicados na literatura apontam concordância com os dados apresentados. Em uma amostra de mães de crianças de quatro meses no Japão, por exemplo, foi constatada uma correlação significativa entre experimentar adversidades socioeconômicas, traduzidas por ter finanças difíceis de manejar ou instáveis, e dois tipos de abuso infantil: sacudir violentamente e abafar a criança (Fujiwara et al., 2016). Na Holanda, entre os fatores de risco sociais para a ocorrência múltipla de TCV, estava a escolaridade cursada até o ensino médio, sendo esta característica presente em $60 \%$ dos pais de crianças vítimas do trauma (Sieswerda-Hoogendoorn et al., 2013). Já no Canadá, alguns especialistas em abuso infantil foram consultados para elencar os fatores que precisam ser mapeados no planejamento de um programa de prevenção ao TCV para direcioná-lo à população que tem maior demanda e, entre tais fatores, estava a baixa renda familiar média (Stewart et al., 2015). Essa maior probabilidade de o trauma acontecer em crianças de famílias mais pobres pode se dever às dificuldades de acesso aos serviços de saúde e às informações necessárias para a prevenção desse problema (Nuño et al., 2015).

Por outro lado, os dados também indicam que famílias com maior poder aquisitivo e maior escolaridade apresentaram, neste estudo, menor conhecimento em relação às características do choro da criança, sendo um fator que aumenta a probabilidade de maus-tratos contra ela. Estes dados também encontram apoio na literatura. Na França, por exemplo, Tursz e Cook (2014) chegaram a resultados mostrando que pais de crianças vítimas de TCV que haviam sido denunciados e julgados pelo poder judiciário eram de classes sociais mais altas e quase metade das mães tinham cursado o ensino superior. Nesse sentido, foram levantadas algumas possibilidades de explicação para o aparecimento dessas correlações. A primeira delas é que é possível que os pais e as mães com maior escolaridade e poder aquisitivo estejam inseridos no mercado de trabalho, consequentemente se afastando do ambiente doméstico por várias horas do dia, permanecendo, assim, menor quantidade de tempo em casa e com o bebê, convivendo menos com o choro infantil e o deixando sob os cuidados de outros familiares, babás e creches; dessa forma, a menor convivência com o choro do bebê pode ter como consequência menor conhecimento a respeito de suas características. Também é possível que mães e pais de maior escolaridade e poder aquisitivo tenham acesso a mais informações de fontes diversas, se comparado com aqueles de menor escolaridade e poder aquisitivo; as informações podem ser advindas de outros familiares, de profissionais de educação, profissionais de saúde, revistas ou livros, podendo ser conflitantes entre si, gerando maiores dúvidas e, consequentemente, inconsistência no momento de responder aos instrumentos.

Também é possível observar, de acordo com os dados expostos, que houve uma relação negativa entre a quantidade de filhos e a utilização de estratégias para manejar o estresse decorrente do choro da criança. É conhecido na literatura que famílias grandes, com maior número de filhos, se encontram entre a população de risco para a ocorrência de formas de violência diversas, como a física e a negligência (Almuneef et al., 2016). Visto que o trauma é uma forma de violência, este fator também está presente entre aqueles que aumentam os riscos de uma criança sofrê-lo. Em um estudo preliminar na Turquia, por exemplo, foi identificado que, entre os fatores de risco presentes nas famílias de crianças com sinais e sintomas indicativos de TCV registrados em prontuários, estava a existência de três ou mais crianças menores de sete anos (Yaylaci et al., 2016). Ou seja, é possível que um número maior de crianças dificulte a disponibilização de tempo para se engajar em atividades que reduzam o estresse advindo dos cuidados dispendidos com os filhos e, assim, aumente a probabilidade de emitir comportamentos violentos contra a criança, como a sacudida violenta, que tem por consequência o TCV.

Observou-se também que, quanto maior a rigidez, menor é o escore total na Escala de Atitudes Frente ao Choro, menores são os conhecimentos em relação às consequências de sacudir o bebê e mais inadequadas são as crenças em relação aos cuidados com a criança. A rigidez é definida pelo manual do CAP como uma inflexibilidade em relação ao comportamento e à aparência da criança (Milner, 1986), no sentido de desejar que a criança esteja sempre limpa e 
nunca desobedeça (Bérgamo et al., 2009). Segundo Bérgamo et al. (2009), os itens dessa subescala se relacionam com um risco maior de maus-tratos. Ainda em relação a essa subescala, ao comparar dois grupos, o clínico (composto por famílias que haviam sido notificadas pelo Conselho Tutelar por situações de abuso físico) e o de comparação (famílias selecionadas em escolas públicas municipais), Patrian et al. (2013) obtiveram como resultados que ambos os grupos apresentaram escore mais alto se comparado com os escores das outras subescalas do instrumento. Isso pode indicar que os pais brasileiros possuem percepções mais rígidas em relação aos seus filhos (Patrian et al., 2013).

Além disso, a rigidez geralmente está presente em pais controladores, autoritários e com maiores expectativas em relação ao desempenho da criança (Cecconello et al., 2003). Nesse sentido, os resultados de Ricci et al. (2003), a partir da avaliação de crianças admitidas em hospital por $\mathrm{TCV}$, apontam que, em $42 \%$ dos casos, estavam presentes expectativas irreais em relação à criança conseguir controlar seu próprio comportamento, compondo, assim, os variados fatores de risco para o trauma ser perpetrado.

Por fim, foi encontrada uma correlação inusitada: a infelicidade está positivamente relacionada aos conhecimentos a respeito das características do choro da criança. Isso quer dizer que, de acordo com os testes estatísticos feitos para esta amostra, a infelicidade está relacionada a maiores conhecimentos sobre o choro. Trata-se de uma correlação inusitada porque, de um lado, os itens de infelicidade avaliados no CAP indicam maior potencial de maus-tratos do respondente (Milner, 1986) e, de outro, maiores conhecimentos a respeito dos padrões e características do choro infantil colaborariam para a redução de comportamentos violentos direcionados ao bebê, como sacudi-lo (Barr, 2014). Portanto, seria uma correlação contraditória.

É possível que essa contradição se deva a algumas fragilidades do estudo, as quais serão apresentadas a seguir. Em primeiro lugar, o estudo contou com amostra pequena, por conveniência, não randômica, o que torna mais difícil a generalização dos dados para outras populações. Além disso, a aplicação dos instrumentos foi feita na presença de outras pessoas, o que pode diminuir o número de respostas correspondentes às opiniões e comportamentos do respondente e aumentar o número de respostas julgadas socialmente aceitas. Assim, sugere-se mais estudos com outras populações, com amostras randômicas, com maior número de participantes, bem como a coleta de dados em locais mais bem controlados e com um menor número de interferências externas.

Os instrumentos utilizados também merecem atenção. Entre eles, a Escala de Atitudes Frente ao Choro do Bebê obteve evidências de validade baseada na sua estrutura interna em um estudo robusto com 500 pais brasileiros de vários municípios do estado de São Paulo, a partir de coleta online e presencial (Lopes, 2017). No entanto, ainda é um instrumento recente e em processo de validação, sendo uma das contribuições desta pesquisa complementar este processo com os dados encontrados.

O CAP, por outro lado, é mais consolidado na área, com vários estudos de validação (Rios, 2010; Rios et al., 2013; Patrian et al., 2013). No entanto, se trata de um instrumento longo, com 160 itens cujas frases nem sempre ficam claras, principalmente para os respondentes com maior dificuldade de compreensão. Para a sua aplicação, é demandado um tempo entre 20 minutos e 60 minutos, a depender da dificuldade que o participante tem de entender o que lhe está sendo perguntado e também da sua vontade de contar histórias a respeito daqueles itens ou se justificar ao longo da aplicação. Tudo isso torna o seu preenchimento cansativo, tanto para quem aplica o instrumento quanto para quem o responde, o que pode ter tido alguma influência sobre os dados aqui encontrados.

Apesar das dificuldades, a aplicação em modelo de entrevista, tal como utilizada na presente pesquisa, pode diminuir as respostas aleatórias, já que $100 \%$ desta amostra teve índice de distorção considerado normal; ou seja, as respostas randômicas, mentiras e inconsistências não ultrapassaram o valor limite.

Para estudos futuros, sugere-se investigar os conhecimentos sobre as consequências de sacudir uma criança e habilidades para lidar com o choro do bebê de outros cuidadores, como os demais familiares (avós, irmãos, padrastos, madrastas), babás e os profissionais de creches e escolas de educação infantil. Essa população merece atenção, uma vez que há uma hipótese de que muitas crianças fiquem sob os cuidados de outras pessoas enquanto os pais desempenham suas atividades laborais fora de casa. Ao mesmo tempo, estudos indicam que, apesar de menos frequentes, quando os perpetradores são pessoas de fora da família, as sequelas do trauma tendem a ser mais severas (Scribano et al., 2013; Schnitzer \& Ewigman, 2005; Nuño et al., 2015). Além disso, parece importante realizar outros estudos de validação da Escala de Atitudes Frente ao Choro com outras populações.

\section{CONSIDERAÇÕES FINAIS}

De modo geral, os dados aqui apresentados indicam que algumas variáveis da Escala de Atitudes Frente ao Choro do Bebê estão mais relacionadas a classes socioeconômicas mais baixas, com renda e escolaridade menores, como as crenças inadequadas, ao passo que outras variáveis estão mais relacionadas com classes socioeconômicas mais altas, como os conhecimentos a respeito do padrão de choro da criança. Isso quer dizer que as intervenções precisam 
ser direcionadas às necessidades de cada população, focando nos aspectos que são mais deficitários para cada população alvo. Ao mesmo tempo, é necessário trabalhar a flexibilidade dos pais nas expectativas que são depositadas nos comportamentos e aparência da criança, assim como as expectativas no seu próprio desempenho parental. Dessa forma, é possível caminhar em direção a intervenções mais efetivas e, consequentemente, à redução das taxas de TCV e das consequências negativas para a saúde e desenvolvimento da criança.

\section{REFERÊNCIAS}

Almuneef, M. A., Alghamdi, L. A., \& Saleheen, H. M. (2016). Family profile of victims of child abuse and neglect in the Kingdom of Saudi Arabia. Saudi Medical Journal, 37(8), 882888. https://doi.org/10.15537/smj.2016.8.14654

Barr, R. G. (2014). Crying as a trigger for abusive head trauma: a key to prevention. Pediatric Radiology, 44(4), 559-564. https:// doi.org/10.1007/s00247-014-3100-3

Bérgamo, L. P. D., Pasian, S. R., Mello, I. L. M. A., \& Bazon, M. R. (2009). O Inventário de Potencial de Abuso Infantil: estudo de precisão e validade. Avaliação Psicológica, 8(3), 425-435. http://pepsic.bvsalud.org/pdf/avp/v8n3/v8n3a14.pdf

Cecconello, A. M., Antoni, C., \& Koller, S. H. (2003). Práticas educativas, estilos parentais e abuso físico no contexto familiar. Psicologia em Estudo, 8(spe), 45-54. https://doi.org/10.1590/ S1413-73722003000300007

Chevignard, M. P., \& Lind, K. (2014). Long-term outcome of abusive head trauma. Pediatric Radiology, 44(4), 548-558. https://doi.org/10.1007/s00247-014-3169-8

Efrom, B. (1980) The Jacknife, the Bootstrap, and other resampling plans (Technical Report $\mathrm{n}^{\circ} 63$ ). Standford University, California.

Fujiwara, T., Yamaoka, Y., \& Morisaki, N. (2016). Self-reported prevalence and risk factors for shaking and smothering among mothers of 4-month-old infants in Japan. Journal of Epidemiology, 26(1), 4-13. https://doi.org/10.2188/jea. JE20140216

Guttman, L. (1945). A basis for analyzing test-retest reliability. Psychometrika, 10(4), 255-282. http://doi.org/10.10007/ BF02288892

Harden, B. J., Buhler, A., \& Parra, L. J. (2016). Maltreatment in infancy: a developmental perspective on prevention and intervention. Trauma, Violence \& Abuse, 17(4), 366-386. https://doi.org/10.1177/1524838016658878

Isumi, A., \& Fujiwara, T. (2016). Association of adverse childhood experiences with shaking and smothering behaviors among Japanese caregivers. Child Abuse \& Neglect, 57, 12-20. https:// doi.org/10.1016/j.chiabu.2016.05.002

Kelly, P., Thompson, J. M. D., Koh, J., Ameratunga, S., Jelleyman, T., Percival, T. M., Elder, H., \& Mitchell, E. A. (2017). Perinatal risk and protective factors for Pediatric Abusive Head Trauma: a multicenter case-control study. Journal Pediatrics, 187, 240246. https://doi.org/10.1016/j.jpeds.2017.04.058

Kemp, M. A. (2011). Abusive head trauma: recognition and the essential investigation. Archive of Disease in Childhood: Education and Practice, 96, 202-208. https://doi.org/10.1136/ adc. 2009.170449

Kuder, G. F., \& Richardson, M. W. (1937). The theory of the estimation of test reliability. Psychometrika, 2, 151-160. https:// doi.org/10/1007/BF02288391

Leetch, A. M., Leipsic, J., \& Woolridge, D. P. (2015). Evaluation of child maltreatment in the emergency department setting: an overview for behavioral health providers. Child \& Adolescent Psychiatric Clinics of North America, 24, 41-64. https://doi. org/10.1016/j.chc.2014.09.006

Lopes, N. R. L., \& Williams, S. C. A. (2016). Avaliação do conhecimento do Trauma Craniano Violento por pais brasileiros. Psicologia: Teoria e Pesquisa, 32(2), 1-11. https:// doi.org/10.1590/0102-3772e32223 e32223

Lopes, N. R. L., Eisenstein, E., \& Williams, L. C. A. (2013). Abusive head trauma in children: a literature review. Jornal de Pediatria, 89(5), 426-433. https://doi.org/10.1016/j. jped.2013.01.011

Lopes, N. R. L. (2017) Trauma craniano violento pediátrico: estratégias de avaliação e prevenção (tese de doutorado). Universidade Federal de São Carlos, São Paulo, Brasil.

Milner, J. S. (1986). The Child Abuse Potential Inventory: Manual (2nd ed.). Webster, NC: Psytec.

Molnar, B. E., Goerge, R. M., Gilsanz, P., Hill, A. Subramanian, S. V., Holton, J. K., Duncan, D. T., Beatriz, E. D., \& Beardslee W. R. (2016). Neighborhood-level social processes and substantiated cases of child maltreatment. Child Abuse \& Neglect, 51, 41-53. https://doi.org/10.1016/j.chiabu.2015.11.007

Nuño, M., Pelissier, L., Varshneya, K., Adamo, M. A., \& Drazin, D. (2015). Outcomes and factors associated with infant abusive head trauma in the US. Journal of Neurosurgery: Pediatrics, 16, 515-522. https://doi.org/10.3171/2015.3.PEDS14544

Parks, S. E., Kegler, S. R., Annest, J. L., \& Mercy, J. A. (2012). Characteristics of fatal abusive head trauma among children in the USA: 2003-2007: an application of the CDC operational case definition to national vital statistics data. Injury Prevention, 18, 193-199. https://doi.org/10.1136/injuryprev-2011-040128

Patrian, A. C. A., Rios, K. S. A., \& Williams, L. C. A. (2013). Validade de critério do Inventário de Potencial de Abuso Infantil (CAP). Paidéia, 23(54), 43-51. https://doi.org/http:// dx.doi.org/10.1590/1982-43272354201306

Piñón, E. A. (2008). Os maus-tratos de crianças e adolescentes na comunidade: o potencial de risco e os fatores sóciocomunitários associados (dissertação de mestrado). Universidade de São Paulo, São Paulo, Brasil.

Ricci, L., Giantris, A., Merriam, P., Hodge, S., \& Doyle, T. (2003). Abusive Head Trauma in Maine infants: medical, child, protective, and law enforcement analysis. Child Abuse \& Neglect, 27, 271-283. https://doi.org/10.1016/S01452134(03)00006-1

Rios, K. S. A. (2010). Inventário de Potencial de Abuso InfantilCAP: adaptação transcultural, fidedignidade e validade para o Brasil (tese de doutorado). Universidade de São Carlos, São Paulo, Brasil.

Rios, K. S. A., Williams, L. C. A., Schelini, P. W., Bazon, M. R., \& Piñón, E. A. (2013). Inventário de Potencial de Abuso Infantil - CAP: evidências de validade e precisão. Avaliação Psicológica, 12(1), 51-60. http://pepsic.bvsalud.org/pdf/avp/ $\mathrm{v} 12 \mathrm{n} 1 / \mathrm{v} 12 \mathrm{n} 1 \mathrm{a} 08 . \mathrm{pdf}$

Schnitzer, P. G., \& Ewigman, B. G. (2005). Child deaths resulting from inflicted injuries: household risk factors and perpetrator characteristics. Pediatrics, 116(5), e687-e693. https://doi. org/10.1542/peds.2005-0296

Scribano, P. V., Makoroff, K. L., Feldman, K. W., \& Berger, R. P. (2013). Association of perpetrator relationship to abusive head trauma clinical outcomes. Child Abuse \& Neglect, 37, 771-777. https://doi.org/10.1016/j.chiabu.2013.04.011 
Selassie, A. W., Borg, K., Busch, C., \& Russel, S. (2013). Abusive Head Trauma in Young children: a population based-study. Pediatric Emergency Care, 29(3), 283-291.

https://doi.org/10.1097/PEC.0b013e31828503ea

Sieswerda-Hoogendoorn, T., Bilo, R. A. C., Duurling, L. L. B. M. V., Karts, W. A., Maaskant, J. M., Aalderen, W. M. C. V., \& Rijn, R. R. V. (2013). Abusive head trauma in young children in the Netherlands: evidence for multiple incidents of abuse. Foundation Acta Paediatrica, 102, e497-e501. https://doi. org/10.1111/apa.12377

Stewart, T. C., Gilliland, J., Parry, N. G., \& Fraser, D. D. (2015). An evidence-based method for targeting an abusive head trauma prevention media campaign and its evaluation. Journal of Trauma and Acute Care Surgery, 79(5), 748-755. https://doi. org/10.1097/TA.0000000000000828

Tanoue, K., \& Matsui, K. (2015). Difference between abusive head trauma and physical abuse in Japan. Pediatrics International, 57, 845-848. https://doi.org/10.1111/ped.12632
Tursz, A., \& Cook, J. M. (2014). Epidemiological data on shaken baby syndrome in France using judicial sources. Pediatric Radiology, 44(4), 641-646. https://doi.org/10.1007/s00247014-3097-7

Yamada, F., \& Fujiwara, T. (2014). Prevalence of self-reported shaking and smothering and their associations with co-sleeping among 4-month-old infants in Japan. International Journal of Environmental Research and Public Health, 11, 6485-6493. https://doi.org/10.3390/ijerph110606485

Yaylaci, S., Dallar, Y., Sayar, Y., Tasar, M. A., Tiras, U., Tekin, D., Unlu, A., Ulukol, B., Beyaztas, F. Y., Butun, C., Ozum, U., Büken, B., Kandemir, F., Gokoglu, A., Kondolot, M., Menku, A., Patiroglu, T., Tunç, A., Yagmur, F., \& Karagoz Guzey, F. (2016). Abusive Head Trauma in Turkey and Impact of Multidisciplinary Team Establishment Efforts on Case Finding and Management: Preliminary Findings. Eurasian Journal of Emergency Medicine, 15, 24-29. https://doi.org/10.5152/ eajem.2016.52386 